\title{
Redox equilibria of iron oxides in aqueous-based magnetite dispersions: Effect of $\mathrm{pH}$ and redox potential
}

\author{
Suh Cem Pang a,*, Suk Fun Chin ${ }^{a}$, Marc A. Anderson ${ }^{b}$

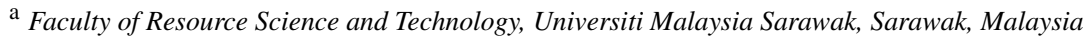 \\ ${ }^{\mathrm{b}}$ Environmental Chemistry and Technology Program, University of Wisconsin-Madison, Madison, WI, USA
}

Received 8 December 2006; accepted 19 February 2007

Available online 28 March 2007

\begin{abstract}
The effect of $\mathrm{pH}$ and redox potential on the redox equilibria of iron oxides in aqueous-based magnetite dispersions was investigated. The ionic activities of each dissolved iron species in equilibrium with magnetite nanoparticles were determined and contoured within the $E_{\mathrm{h}}-\mathrm{pH}$ framework of a composite stability diagram. Both standard redox potentials and equilibrium constants for all major iron oxide redox equilibria in magnetite dispersions were found to differ from values reported for noncolloidal systems. The "triple point" position of redox equilibrium among Fe(II) ions, magnetite, and hematite shifted to a higher standard redox potential and an equilibrium constant which was several orders of magnitude higher. The predominant area of magnetite stability was enlarged to cover a wider range of both $\mathrm{pH}$ and redox potentials as compared to that of a noncolloidal magnetite system.
\end{abstract}

(C) 2007 Elsevier Inc. All rights reserved.

Keywords: Magnetite; Dispersion; Nanoparticles; Redox potential; Redox equilibria

\section{Introduction}

Magnetite $\left(\mathrm{Fe}_{3} \mathrm{O}_{4}\right)$ is a ferrite which possesses an inverse spinel structure. Nanosized magnetite particles have long been of technological interests due to their utility in magnetic recording media, protective and sensitive coatings, as catalysts and pigments, and various technological applications. Intensive research efforts have been focused on the development of appropriate protocols for preparing magnetite nanoparticles with well-defined uniform size and shape in a reproducible manner [1-7]. Magnetite is hydrophilic in nature, and it is negatively and positively charged nanoparticles in the alkaline and acidic medium, respectively. As a consequence of their large surface area to volume ratios, nanosized magnetite particles are inherently unstable with a high tendency to aggregate in order to minimize their high surface energies. Although magnetite nanoparticles can be easily prepared by coprecipitation of $\mathrm{Fe}(\mathrm{II})$ and $\mathrm{Fe}$ (II) ions in an alkaline solution, judicious choice of

\footnotetext{
${ }^{*}$ Corresponding author. Fax: +60 82583160.

E-mail address: scpang@ frst.unimas.my (S.C. Pang).
}

solvent and coating agent for surface functionalization of magnetite particles is crucial in preventing particle aggregation and to ensure the formation of magnetite nanoparticles with uniform sizes. Obviously, the key technological challenges in the synthesis of superparamagnetic magnetite nanoparticles are the ability to precisely control their particle sizes, dispersibility, and stability in the desired solvent media. For instance, the efficacy of superparamagnetic materials for various biomedical applications has been shown to depend strongly on the synthesis of magnetite nanoparticles with narrow size distribution and that are well-dispersed in aqueous medium [8-11].

Magnetite is one of the most common forms of iron oxides. In general, metal oxides are known to undergo complex interfacial charging processes which involve $\mathrm{H}^{+}$and $\mathrm{OH}^{-}$ions as potential determining ions when they are in contact with aqueous electrolytes [12-26]. An electrically charged interface is developed with the adsorption of $\mathrm{H}^{+}$or $\mathrm{OH}^{-}$ions through the protonation and deprotonation of amphoteric surface groups according to

$$
\begin{aligned}
& \mathrm{SOH}^{0}+\mathrm{H}^{+} \leftrightarrow \mathrm{SOH}_{2}^{+}, \\
& \mathrm{SOH}^{0} \leftrightarrow \mathrm{SO}^{-}+\mathrm{H}^{+},
\end{aligned}
$$

